\title{
E33 FILTRATION-FLOW EFFECTS AND VARIANTS OF CONSTRUCTING NONLINEAR FILTRATION LAWS IN THE CASE OF VIOLATION OF THE DARCY LAW WITH ISOTROPIC FILTRATION PROPERTIES
}

V.M. Maksimov ${ }^{1}$ and N.M. Dmitriev ${ }^{2}$

${ }^{1}$ Research Institute Oil and Gas of RAS, Russia

${ }^{2}$ Gubkin State University of Oil and Gas, Russia

\section{Introduction}

Numerous experimental studies have established not only the possibility of using the Darcy law but also limits of its applicability. In the process of these investigations, the Darcy law was shown to be valid for both isotropic and anisotropic media, but it is fulfilled merely within a certain range of flow velocities. Thus, the upper and lower boundaries for the applicability of the Darcy law can be indicated. The upper boundary is caused by the manifestation of inertial forces at high velocities, while the lower one, by physicochemical effects for interaction of a fluid with a solid skeleton and by non-Newtonian rheological properties of the fluid. In this study, we consider the generalizations of the Darcy law with isotropic filtration properties at high filtration rates.

A number of papers [1,2] are devoted to constructing nonlinear constitutive equations of the filtration theory at high filtration rates. However, in these investigations, methods of the theory of nonlinear tensor functions and those of crystal physics $[3,4]$ were hardly used. Using the theories indicated makes it possible not only to develop general methods of constructing nonlinear constitutive equations but also to find possible effects. Analysis of nonlinear filtration laws generalizing the Darcy law with isotropic filtration properties showed that nonlinear filtration properties can be anisotropic and, moreover, manifest asymmetry; i.e., they have different filtration properties in the case of flow along the same straight line in different directions.

\section{Basic postulates and formulas}

The basic law of the filtration theory (Darcy rule) represents the linear relation between two effective vector fields, namely, the filtration-pressure gradient $\nabla_{i} p$ and the filtration rate $w_{i}$. The presence of this relation is experimental fact. On the other hand, the Darcy rule can be derived by averaging the Navier - Stokes equations for the case of the fluid flow in a periodic lattice [5]. Thus, the Darcy rule represents the differential form of the momentum-conservation law for a specific continuous medium that represents a composite material consisting of solid skeleton and a fluid. Therefore, in the Darcy rule, both fluid properties and void-space properties of the solid skeleton are given and specified. In the case of filtration of a Newtonian viscous fluid, it turns out that these properties can be separated. The fluid properties are specified only by the viscosity coefficient $\mu$ while the void-space properties are determined by its symmetry and can be given by the penetrability-coefficient tensor $k_{i j}$ or by the tensor $r_{i j}$ for the, filtrationresistance coefficients. The last argument makes the Darcy rule similar to constitutive 
relationships like Ohm's or Fick's laws. This is associated with the fact that one more property ойле fluid motion is introduced by the Darcy rule.

In the context of constitutive relationships, the principal equation of the filtration theory postulates, in the general case, a relation of the form

$$
\nabla_{i} p=f_{i}\left(w_{i}, \rho, \mu, \chi_{\alpha}, T_{\alpha}\right)
$$

where $\rho$ is the fluid density, $\chi_{\alpha}$ are the invariant scalar parameters describing a porous medium and possibly the fluid, and $T_{\alpha}$ are the material tensors specifying and setting the properties of the void space for the solid skeleton. Assumption of the linearity for relation (1) leads to the Darcy rule. The simplest variant for generalizing the Darcy rule, which corresponds to equation (1), implies the subsequent expansion of the functions $f_{i}$ into the Taylor series in terms of powers of $w_{i}$ :

$$
\frac{1}{\mu} \nabla_{i} p=-r_{i j} w_{j}-r_{i j k} w_{i} w_{k}-r_{i j k l} w_{j} w_{i} w_{l}
$$

Here $r_{i j}, r_{i j k}$ and $r_{i j k l}$ are the material tensors setting the filtration resistance. In equality (2) and below, we consider the problem in the Cartesian coordinate system and assume, for simplicity, that the summation is carried out with respect to the repeated subscripts. The explicit forms of the tensors $r_{i j}, r_{i j k}$ and $r_{i j k l}$ depend on the class (group) of symmetry of the void space $[3,4]$. For an isotropic porous medium, relationship (2) yields the filtration law in the form

$$
\frac{1}{\mu} \nabla_{i} p=-r_{1} w_{i}-r_{2} w^{2} w_{i}
$$

where $r_{i}$ are the scalar coefficients and $w$ is the modulus of the filtration-rate vector. Relationship (3) differs from the Forchheimer law [2], commonly used in underground hydromechanics, in which the nonlinearity is given by a term of the form $r_{2} w w_{i}$. It is necessary to note that the result presented by relationship (3) was found previously in [6] as the solution to the Ozeen and Navier - Stokes equations. To substantiate the Forchheimer formula and obtain a more general representation for relations (1), it is necessary to use the more rigorous results of $[7,8]$. According to these studies, relations (1) can be represented in the form of the expansion in terms of the basis tensors and their combinations [8], whose multipliers are functions of the invariants (the generalized Hamilton Cayley formulas). In this case, the representation of the most general form of the nonlinear filtration law for isotropic porous media is given by the relationship [7]

$$
\frac{1}{\mu} \nabla_{i} p=f g_{i j} w_{j}
$$

where $f$ is the function of $w$ and $g_{i j}$ is the metric tensor. The construction of filtration law (4), i.e., the definition of the explicit form of $f(w)$ for a particular isotropic porous medium can be realized by processing only one experimental curve representing the dependence of the 
gradient-pressure modulus on the modulus of the filtration-rate vector. Indeed, it follows from relationship (4) that $f(w)=\nabla p / \mu w$. Thus, the approximation of the experimental dependence of $\nabla p$ on $w$ determines the form of $f(w)$ and, therefore, the nonlinear filtration law. In the framework of a such approach, the one-term and two-term representations of the nonlinear filtration law [2] differ only by the choice of the class of functions used for the approximation. However, the isotropic filtration properties in the Darcy rule are manifested also by anisotropic porous media with the cubic symmetry of the effective void space [4]. Porous media with a cubic symmetry of the effective void space have the following generalized representations for the nonlinear laws accurate to the cubic terms in the filtration-rate expansion [8]:

$$
\begin{gathered}
\frac{1}{\mu} \nabla_{i} p=-f_{1} g_{i j} w_{j}-f_{2} O_{(h) i j k l} w_{j} w_{k} w_{l}, \\
\frac{1}{\mu} \nabla_{i} p=-\varphi_{1} g_{i j} w_{j}-\varphi_{2} T_{(d) i j k} w_{j} w_{k}-\varphi_{3} O_{(h) i j k l} w_{j} w_{k} w_{l}
\end{gathered}
$$

where $f_{i}$ and $\varphi_{i}$ are the functions of the main vector invariants formed by convolutions of basis tensors with the filtration-rate vector, $g_{i j}, T_{(d) i j k}$ and $O_{(h) i j k l}$ are the basis tensors [8]. Equality (5) yields the filtration law for the groups (classes) of symmetry $\overline{6} / 4, \overline{3} / 4, \overline{6} / 2$, and equality (6), $3 / \overline{4}$ and $3 / 2$ (the notation for the symmetry groups is given according to Shubnikov). The analysis of relationships (4) - (6) shows that they all represent the Darcy rule with isotropic filtration properties in the case when $f, f_{1}$ and $\varphi_{1}$ are constant and $f_{2}=0$, $\varphi_{2}=0$, and $\varphi_{3}=0$. However, while passing to the nonlinear filtration law, the filtration properties substantially differ. The nonlinear filtration resistances specified by the relationship $r\left(n_{i}\right)=-\nabla_{i} p n_{i} / \mu w$ have the following form for filtration laws (4), (5), and (6), respectively:

$$
\begin{gathered}
r=f(w), \quad r=f_{1}+f_{2}\left(n_{1}^{4}+n_{2}^{4}+n_{3}^{4}\right) w^{2}, \\
r=\varphi_{1}+6 \varphi_{2} n_{1} n_{2} n_{3} w+\varphi_{3}\left(n_{1}^{4}+n_{2}^{4}+n_{3}^{4}\right) w^{2}
\end{gathered}
$$

where $n_{i}$ are the components of the basis vector determining the direction of the filtration-rate vector with respect to the crystallographic axes [8]. Analysis of expressions (7) shows that the filtration properties in laws (5) and (6) are direction-dependent and, moreover, they manifest an asymmetry in law (6) along the direction for which $n_{1} n_{2} n_{3} \neq 0$. Thus, a change in the symmetry of filtration properties is possible while passing from the linear to the nonlinear filtration law. It is necessary to take this fact into account in the experimental determination of these laws. Therefore, when constructing the nonlinear filtration laws, we need to carry out complex experimental investigations even for media manifesting isotropic properties in the Darcy rule.

\section{On experimental determining the coefficients in nonlinear relations}


The procedure of experimentally determining nonlinear filtration properties for actual rocks containing hydrocarbon row material must include determination of both the effective symmetry of the void space and the explicit form of the functions $f_{i}$ and $\varphi_{i}$. One variant for complex investigations can involve determination of elastic and hydrodynamic effective properties. Using the elastic characteristics of a sample, it is possible to determine the symmetry of elastic properties, which coincides with the symmetry of the fourth-rank tensor in representations (2), (5), and (6), and to establish the directions of crystallographic axes [9, 10]. After the elastic symmetry and positions of main axes have been determined, it is necessary to cut several smaller samples from the primary one for subsequent hydrodynamic studies. In the case of cubic symmetry, the minimum number of samples can be two for relationships (5) and three for relationships (6). However, since the elastic symmetry of cubic crystals is given by the tensor $O_{(h) i j k l}$ for all symmetry groups, it is impossible to determine the type of the nonlinear filtration law after establishing only elastic symmetry. Therefore, it is necessary to prepare samples. The optimum directions of the sample symmetry axes are the following:

$$
n_{i}^{(1)}=(1,0,0), n_{i}^{(2)}=\left(\frac{\sqrt{2}}{2}, \frac{\sqrt{2}}{2}, 0\right), n_{i}^{(3)}=\left(\frac{\sqrt{3}}{3}, \frac{\sqrt{3}}{3}, \frac{\sqrt{3}}{3}\right) .
$$

As is easy to establish for the directions found, the vectors $w_{i}$ and $\nabla_{i} p$ are located on one straight line and, as a result of experimental investigations, the plots of the dependences $\Delta p / L=F\left(w, n_{i}^{(\alpha)}\right)$ are obtained, where $\Delta p$ is the difference of pressures or the Leibenzon functions on the sample in the course of the fluid and gas filtration, respectively. Furthermore, $L$ is the sample length, and $w$ и is the filtration rate for the fluid filtration or the mass-flow rate for the gas filtration. Expressing experimental data in the form

$$
\frac{\Delta p}{\mu w L}=\frac{F\left(w, n_{i}^{(\alpha)}\right)}{\mu w}
$$

we find the values of the filtration resistances $r\left(n_{i}^{(\alpha)}\right)$ along the corresponding directions. As has been noted, in the general case, the desired expressions for $f_{i}$ and $\varphi_{i}$, are the functions of the invariants formed by convolutions of the rate vector with the basis tensors. However, the assumption that $f_{2}, \varphi_{2}$ and $\varphi_{3}$ depend on the invariants increases the exponent for the rate in the representation of the filtration resistances (7). This considerably complicates the representation for these dependences, whereas all the known experimental data are well processed using two or three constants in the polynomial representation of the filtration law. Therefore, remaining within the framework of the classical representation of the nonlinear filtration law, we can assume that $f_{1}=\varphi_{1}=a+b w, \varphi_{2}=c, f_{2}=\varphi_{3}=d$, where $a$, $b, c$, and $d$ are certain constants to be found experimentally. In this case, the linear combinations of the filtration resistances $r\left(n_{i}^{(\alpha)}\right)$ for the directions $n_{i}^{(1)}$ and $n_{i}^{(2)}$ make it possible to determine $d$ :

$$
r\left(n_{i}^{(1)}\right)-r\left(n_{i}^{(2)}\right)=0,5 d w^{2}
$$


and $a+b w$ :

$$
2 r\left(n_{i}^{(2)}\right)-r\left(n_{i}^{(1)}\right)=a+b w .
$$

The representation of the constant $C$ in filtration law (6) is obtained from the measurements for the third sample along the positive $+n_{i}^{(3)}$ and the negative $-n_{i}^{(3)}$ directions:

$$
r\left(+n_{i}^{(3)}\right)-r\left(-n_{i}^{(3)}\right)=\frac{4 \sqrt{3}}{3} c
$$

Carrying out the experimental studies according to the scheme proposed and processing the experimental data on the basis of relationships (8) - (10) make it possible to find the nonlinear filtration law generalizing the Darcy rule with isotropic filtration properties. In this case, feasible effects associated with manifestation of the anisotropy and asymmetry in filtration properties are taken into account.

The analysis of constructing nonlinear filtration laws for media manifesting transversal-isotropic and orthotropic filtration properties in the Darcy rule is related to even greater "structuring" of both the filtration properties and filtration laws and presents an object for additional study.

\section{ACKNOWLEDGMENTS}

This work was supported by the Russian Foundation for Basic Research, project no. 00-01-00609.

\section{References}

[1] S. V. Belov, Porous Substances in Industry (Mashinostroenie, Moscow, 1981).

[2] K. S. Basniev, I. N. Kochina, and V. M. Maximov, Underground Hydromechanics (Nedra, Moscow, 1993).

[3] Yu. I. Sirotin and M. P. Shaskol'skaya, Principles of Crystal Physics (Nauka, Moscow, 1975).

[4] V. V. Lokhin and L. I. Sedov, Prikl. Mat. Mekh. 27 (3), 293 (1963).

[5] N. S. Bakhvalov and G. P. Panasenko, Averaging of processes in Periodic Media (Nauka, Moscow, 1984).

[6] Yu. P. Korotaev and M. B. Panfilov, Development of Methods for Determining Parameters of the Data of Its Microstructure (IRTs Gazprom, Moscow, 1993).

[7] Yu. I. Sirotin, Pricl. Mat. Mekh. 28, 653 (1964),

[8] Yu. I. Sirotin and V. F. Pleshakov, Pricl. Mat. Mekh. 30, 243 (1966).

[9] A. M. Kuznetsov, A. G. Kovalev, D. I. Sal'nikov, et al., Neft. Khoz., No 7, 44 (1997).

[10] T. N. Krechetova and E. S. Romm, Izv. Akad. Nauk SSSR, Makh. Zhidk. Gaza, No. 1, 181 (1983). 
